Editorial

\title{
Acknowledgement to Reviewers of Journal of Cardiovascular Development and Disease in 2018
}

\author{
Journal of Cardiovascular Development and Disease Editorial Office
}

MDPI, St. Alban-Anlage 66, 4052 Basel, Switzerland

Published: 10 January 2019

\begin{abstract}
Rigorous peer-review is the corner-stone of high-quality academic publishing. The editorial team greatly appreciates the reviewers who contributed their knowledge and expertise to the journal's editorial process over the past 12 months. In 2018, a total of 58 papers were published in the journal, with a median time to first decision of 14 days and a median time to publication of 36 days. The editors would like to express their sincere gratitude to the following reviewers for their cooperation and dedication in 2018:
\end{abstract}

\author{
Ahmad, Shakil \\ Anderson, Robert \\ Aránega, Amelia \\ Aronow, Wilbert \\ Asirvatham, Samuel J. \\ Ayme-Dietrich, Estelle \\ Ballmann, Christopher \\ Beavo, Joe \\ Belo, José António \\ Billaud, Marie \\ Blaser, Mark \\ Bolea, Juan \\ Borén, Jan \\ Braun, Thomas \\ Broderick, Tom L. \\ Broom, Iain \\ Bucchi, Andrea \\ Burgon, Patrick G. \\ Butlin, Mark \\ Campione, Marina \\ Campos-Toimil, Manuel \\ Cao, Jingli \\ Capoccia, Massimo \\ Cedó, Lídia \\ Ceulemans, Berten \\ Chaudhry, Bill \\ Chen, Yung-Chih \\ Ciccone, Marco Matteo \\ Cinnamon, Yuval \\ Curci, John A. \\ Czubryt, Michael P. \\ De Vecchis, Renato \\ Degenhardt, Karl \\ Dell'Acqua, Mark
}

Dettman, Robert W.

Dimova, Elitsa

Diviani, Dario

Eichstaedt, Christina

Esposito, Francesco

Ezhov, Marat V.

Fedele, Francesco

Fetterman, Jessica L.

Franco, Diego

Genoni, Angela

German, Charles

Gittenberger-De Groot, Adriana C.

Glover, Mark

Grimes, Daniel T.

Gubin, Denis

Guo, Chao-Yu

Gurha, Priyatansh

Hall, Kathryn T.

Harvey, Robert

He, Jia-Qiang

Hebert, Terry

Hiroi, Noboru

Hosick, Peter

Houck, Philip D.

Houston, Mark C.

Huang, Christopher

Ikonomidou, Vasiliki N.

Intapad, Suttira

Ivanovitch, Kenzo D.

Iwasa, Toru

Jadhav, Gopal P.

Janus, Edward

Jaźwińska, Anna

Jeong, In Cheol 
Jeremy, Richmond

Jouk, Pierre Simon

Kang, Junsu

Kardassis, Dimitris

Karra, Ravi

Kassiri, Zamaneh

Kawashima, Tomokazu

Kelly, Robert G.

King, Benjamin

Klussmann, Enno

Kocica, Mladen J.

Koga, Jun-ichiro

Kostner, Gerhard

Kostner, Karam

Kumar, Sandeep

Lang, Di

Lazzeri, Chiara

Lee, Dong Ik

Lefkimmiatis, Konstantinos

Leung, Alan

Levy, Finn Olav

Li, Shuai

Lien, Ching-Ling (Ellen)

Lin, Wey-jinq

Lorenzo González, Óscar

Loughna, Siobhan

Lugnier, Claire

Lui, Kathy O.

Macquaide, Niall

Maiellaro, Isabella

Männer, Jörg

Marín-Juez, Rubén

Maroteaux, Luc

McCoy, Sarah J. Breese

McDaneld, Tara G.

McNamara, Coleen A.

Meens, M.J.P.M.T.

Mohamed, Salah A.

Molina, Cristina E.

Morimoto, Tatsuya

Moriya, Manabu

Movesesian, Matthew

Myasoedova, Veronika

Nakamura, Kazufumi

Naro, Fabio

$\mathrm{Ng}$, Fu Siong

Nikolaev, Viacheslav

Nishigaki, Takuya

O'Neill, Louisa

Pacurari, Maricica

Paterson, David J.
Postma, Alex V.

Prakash, Siddharth K.

Prickaerts, Jos

Protogerou, A.D.

Provazník, Ivo

Puri, Kriti

Rocha, Sonia

Rodríguez-Pérez, José Manuel

Rubart, Michael

Rugonyi, Sandra

Sacconi, Leonardo

Sampietro, Tiziana

Sander, Veronika

Sang, Bing Ong

Santulli, Gaetano

Sanyahumbi, Amy E.

Schmidt, Martina

Sedmera, David

Shewale, Swapnil

Shimada, Yasuhito

Shiratori, Hidetaka

Sinzinger, Helmut

Sivaguru, Mayandi

Slark, Julia

Smyth, James

Song, Su

Staples, Anne

Strychalski, Wanda

Sun, Yunfu

Talman, Virpi

Tanaka, Toshihiro

Tritapepe, Luigi

Verhoeven, Adrie

Ward, Marie-Louise

Watanabe, Eiichi

Watanabe, Michiko

Watts, Gerald

Weninger, Wolfgang J.

Wesson, Don

Winkler, Johannes

Won, Kimberly

Wu, Mingfu

Yamada, Shigehito

Yamagishi, Hiroyuki

Yanagisawa, Satoshi

Yarwood, Stephen

Yeh, Jwu-Lai

Yin, Voot

Yoshimura, Masami

Zoltowska, Dominika M.

(C) 2019 by the author. Licensee MDPI, Basel, Switzerland. This article is an open access article distributed under the terms and conditions of the Creative Commons Attribution (CC BY) license (http://creativecommons.org/licenses/by/4.0/). 